\title{
Computing the Three Dimensional Depth Measurement by the Multi Stereo Images
}

\author{
Sandar Aung
}

\begin{abstract}
The photorealistic constructing 3D models of an object or a scene from images is still a very difficult task in 3D image modelling research area. The reconstruction of a $3 D$ model can have different application in many areas, such as industrial, virtual reality, and movies, entertainment, among others. The depth estimation is occupied the essential role in 3D model reconstruction. The foreground object is extracted by the color segmentation approach. The reference points of an object are automatically selected from the image and compute the perspective projection between $2 \mathrm{D}$ and $3 \mathrm{D}$ relation. The fundamental matrix is constructed by the location of the corresponding pixel in each image. Then $3 \mathrm{D}$ cloud points are computed for obtaining the minimum 3D bounding box of the model. It will provide the $3 \mathrm{D}$ surface reconstruction by integrating the 3D cloud points and relative color information. The multi stereo camera system is applied to get the accurate depth measurements of an object. The effectiveness of the proposed approach can be confirmed through the experimental results.
\end{abstract}

Index Terms-Constructing photorealistic 3D models, 3D model generating system, 3D cloud points, color segmentation, perspective projection, depth measurement.

\section{INTRODUCTION}

The 3D modeling is a useful technology in various application fields such as the entertainment industry, technical visualization, e-commence work, 3D simulation work, robotic technology and 3D communication over the Network. Object reconstruction is a challenging problem in generating a 3D model of an object given a set of 2D images of the object. There are two classes of object reconstruction: active methods_3D model acquisition can be performed by laser scanners or structured light; passive methods reconstruct $3 \mathrm{~d}$ models by image sequences from a single camera or multiple cameras. Object scanning often demands expensive equipments and special skill to operate. As a simple and a low cost approach, 3D object reconstruction based images became more and more popular among the researchers. The accurate depth measurement is essentially required to obtain the good 3D feature.

Many related proposals of 3D depth estimation and 3D reconstruction of the object model have been already proposed [1]-[9]. Lutz Falkenhagen [2] presented an algorithm for estimating reliable and accurate depth maps from stereoscopic image pairs which is based on blockmatching techniques for disparity estimation. Karsten, et al.

Manuscript received April 1, 2013; revised July 14, 2013.

Sandar Aung is with the University of Computer Studies, Mandalay, Myanmar (e-mail: sandaraung84@gmail.com).
[3], described in efficient implimation for correlation based stereo. The dense disparity maps are produced from color stereo images. Masatohi Oktomi et al. proposed the multibaseline stereo method [4]. Frederic Huguet et al. [5] presented a variational method for scene flow Estimation from a calibrated stereo sequence. The dense matching between the images is recovered.

An automatic computing system of the depth measurement of an object and a scene is developed in this research. It will be provided to the 3D model generation of an object. The reference points of image are automatically extracted from the edge image of an object. To determine the correlated feature points of the whole object, the edge feature points of the object shape are estimated firstly and the transformation matrix is built by these corresponding pairs. Then the correlated feature pairs of whole object are obtained by using this transformation matrix. Stereo method is a useful technique for estimating the depth of an object from left and right $2 \mathrm{D}$ images as eyes in human vision system.

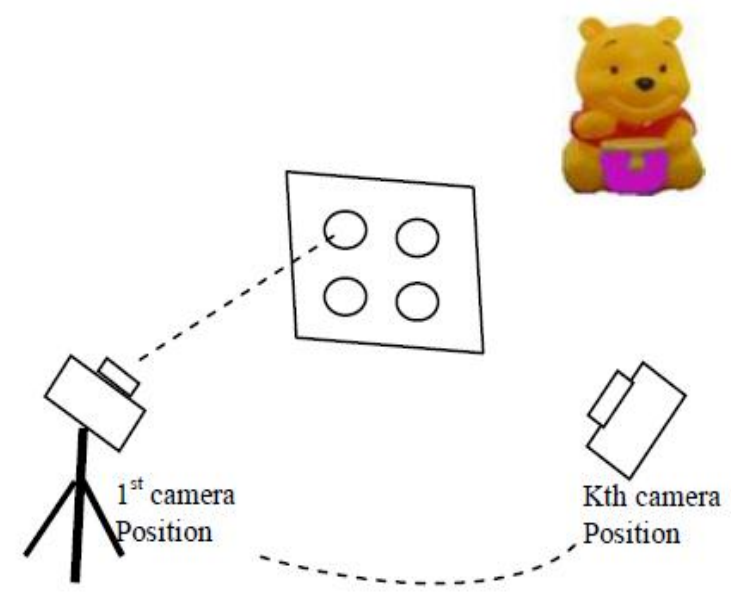

Fig. 1. Illustration of the multi stereo camera system.

Determining the depth measurement from the single stereo pair often has occlusion errors due to the camera alignment. In this research work, for reducing the occlusion errors and ambiguity in matching, the multiple stereo system is developed to generate the depth map. The quadranocular camera system is applied in this research. The six stereo pairs $\left({ }^{4} C_{2}=4.3 / 2=6\right)$ are sufficient to obtain the exact depth measurement of an object. The 3D cloud point's estimation is performed for surface model reconstruction. Multi_Stereo Camera approach will supports for obtaining the more accurate depth measurements of an object than the single stereo image pairs. Fig. 1 illustrated the image multi stereo acquisition system. 
The successive images are taken by changing the camera positions. The camera focal length is $35 \mathrm{~mm}$ and image of object is acquired from $100 \mathrm{~cm}$ apart. The distance between the two camera centers is set to $70 \mathrm{~mm}$. The distance between the object position and camera position is $140 \mathrm{~cm}$. Let the bottom left image, bottom right image, upper left image and upper right image are defined as $I^{(0,0)}, I^{(1,0)}, I^{(0,1)}$ and $I^{(1,1)}$, respectively. The four acquired stereo images are shown in Fig. 2. The three dimensional depth measurements are computed based on the multi stereo camera system for obtaining the exact 3D measurements.

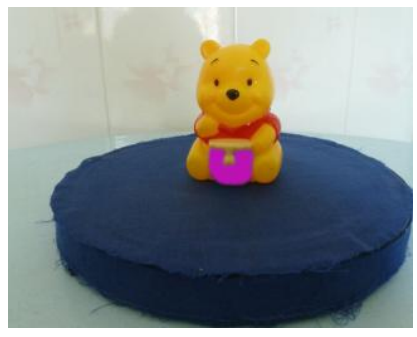

(a) $I^{(0,1)}$

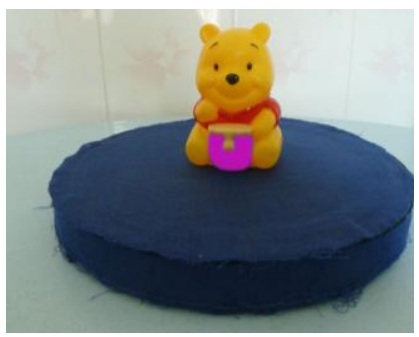

(c) $I^{(0,0)}$

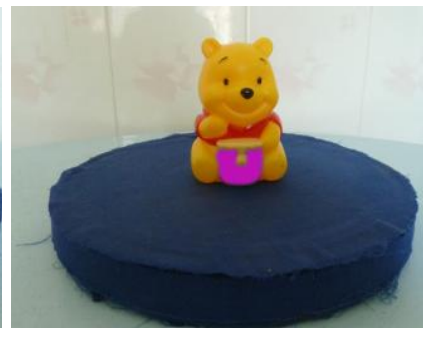

(b) $I^{(1,1)}$

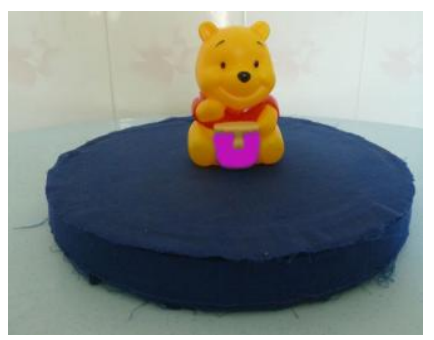

(d) $I^{(1,0)}$
Fig. 2. Acquired images of the quadranocular camera system.

\section{CORRESPONDing RElation AMONG THE STEREO PAIRS}

The perspective projection of a three dimensional point $(X$, $Y, Z$ ) to a two dimensional point on the image plane is express by the camera focal length $\mathrm{f}$,

$$
x=f \frac{X}{Z} \quad, y=f \frac{Y}{Z} .
$$

The point of $p(x, y)$ for the bottom left image $I^{(0,0)}$ can be expressed by equation (1),

$$
I^{(0,0)}(x, y)=I^{(0,0)}\left(f \frac{X}{Z}, f \frac{Y}{Z}\right)
$$

The image point of $p(x, y)$ on other image $I^{(r, s)}$ is also defined as

$$
I^{(r, s)}(x, y)=I^{(r, s)}\left(f \frac{(X-r b)}{Z}, f \frac{(Y-s b)}{Z}\right),
$$

where $b$ is the base line $r$ and $s$ are the row and column distance from the image $I^{(0,0)}$. The disparity $d$ can be expressed by the difference between the corresponding points for two images and depth $Z$ as:

$$
d=\frac{f b}{Z}
$$

Then the corresponding relation of each image can be defined by $I^{(r, s)}(x-r d, y-s d)$.

\section{CORRESPONDING ESTIMATING THE DEPTH MEASUREMENT}

The foreground extraction, edge detection and disparity range computing are performed after image enhancing. The extracted foreground images of image $I^{(0,0)}$ and image $I^{(0,1)}$ are described in Fig. 3(a) and Fig. 3(b), respectively.

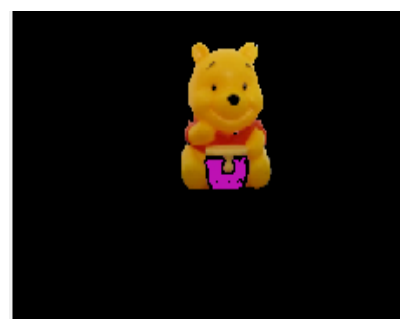

(a)

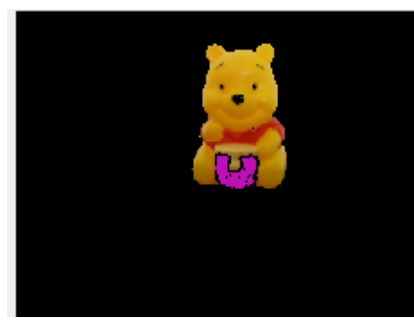

(b)
Fig. 3. Foreground extraction.

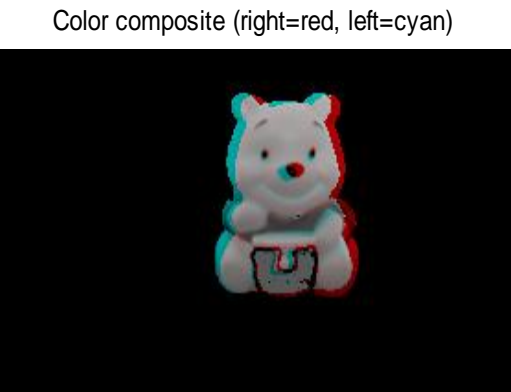

Fig. 4. Composite image.

Their color composite image is shown in Fig. 4. The surface feature points are detected in both images and Fig. 5 (a) is illustrated the auto detected edge feature points in two stereo images. Gaussian correlation based on the singular value decomposition approach is applied for computing the matching pairs between images. The corresponding pairs of an object after geometric correction are illustrated in Fig. 5 (b), respectively.

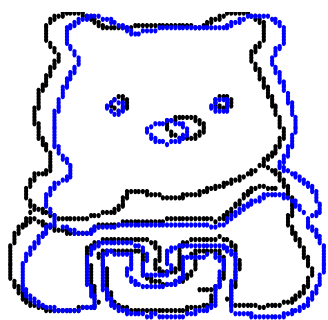

(a)

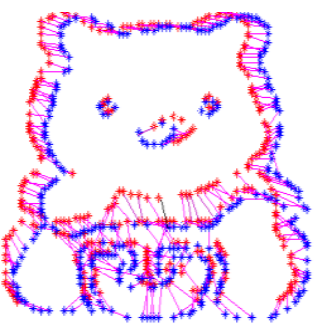

(b)
Fig. 5. Matching of the edge feature points.

Then all corresponding image pairs between two images are computed by the transformation matrix. For the two feature set $\mathrm{S} 1$ and $\mathrm{S} 2$, the transformation matrix $\mathrm{T}$ is computed as follow:

$$
\begin{gathered}
\mathrm{S}_{2}=\mathrm{TS}_{1} \\
\mathrm{~S}_{2}\left(\mathrm{~S}_{1}\right)^{\prime}=\mathrm{TS}_{1} \mathrm{~S}_{1}{ }^{\prime} \\
\mathrm{S}_{2} \mathrm{~S}_{1}{ }^{\prime}\left(\mathrm{S}_{1} \mathrm{~S}_{1}{ }^{\prime}\right)^{-1}=\mathrm{T}\left(\mathrm{S}_{1} \mathrm{~S}_{1}{ }^{\prime}\right)^{*}\left(\mathrm{~S}_{1} \mathrm{~S}_{1}{ }^{\prime}\right)^{-1} \\
\mathrm{~T}=\mathrm{S}_{2} \mathrm{~S}_{1}{ }^{\prime}\left(\mathrm{S}_{1} \mathrm{~S}_{1}{ }^{\prime}\right)^{-1}
\end{gathered}
$$

The whole feature points in whole image are shown in Fig. 6. 


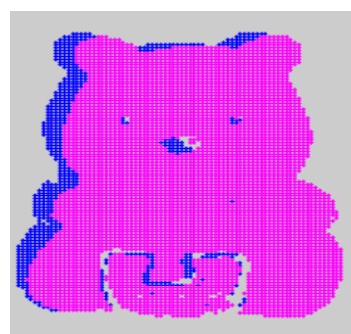

Fig. 6. Matching feature points of whole image.

\section{DEPTH COMPUTING}

Consider the left and right stereo camera system as following Fig. 7. If the positions of the image $P$ in two cameras are $x_{0}$ and $x_{1}$ for focal length $f$, the depth measurement $Z$ can be described as:

$$
Z=\frac{f b}{\left(x_{1}-x_{0}\right)}
$$

where $b=X_{1}-X_{0} ; Z=f b /\left(x_{1}-x_{0}\right)$.

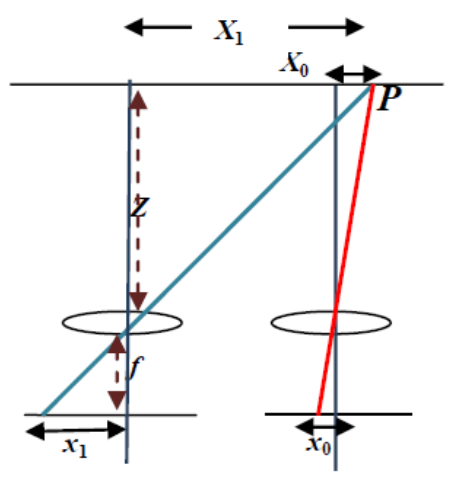

Fig. 7. Depth measuring of stereo system.

\section{EXPERIMENT AL RESULT}

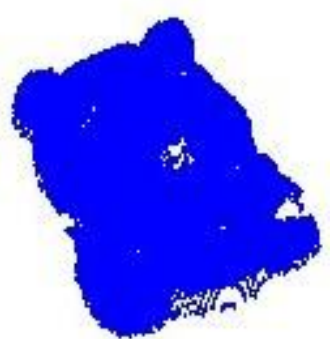

Fig. 8. 3D Cloud Points of an Object.

A pooh doll is used for experiments. More than two images are required for computing the exact depth measurement. The successive images are taken by changing the camera's positions. In this performance, FUGIFILM FinePix F80EXR is applied for image acquisition. The depth measurements of an object are computed by the correlated feature point pairs of six stereo image pairs of an object. The 3D clouded points are obtained by using the depth image. Fig. 8 described the 3D cloud points of an object.

\section{CONCLUSIONS}

In this paper, the depth computation of an object from the multiple images taken by the multi stereo camera system is presented. The correlated feature pairs of the whole image are computed in a short time by the transformation matrix of initial correlated feature pair. The time complexities and cost will reduced by this approach. Through the experimental result, the proposed method works adequately.

\section{REFERENCES}

[1] T. Kanade et al., "Development of a Video-Rate machine," in Proc Int. Robotic and Systems Conf. (IROS '95), Pittsburgh PA., Aug. 1995.

[2] L. Falkenhagen, "Depth estimation from stereoscopic image pairs assuming piecewise continuos surfaces," Euro-pean Workshop on Combined real and synthetic image processing for broadcast and video productions, 2000, Germany.

[3] Karsten et al., "Calculating the dense disparity maps from color stereo images, an efficient implementation," Euro-pean Workshop on Combined real and synthetic image processing for broadcast and video productions, Germany.

[4] M. Oktomi et al., "A multi-baseline stereo," in Proc. Computer Vision and Pattern Recognition, June 1991.

[5] F. Huguet et al., "A variational method for scene flow estimation from stereo sequences," IEEE International Conference ICCV 07, 2007.

[6] R. Szeliski and S.B. Kang, "Recovering 3D shape and motion from image streams using non-linear least squares," Journal of Visual Communication andImage Representation, vol. 5, no. 1, pp. 10-28, 1994.

[7] M. M. Sein and Y. Arakawa, "3D model generating of an object using multiple eyes stereo system," Keihanna Human Info-Communications Research Center, CRL, Kyoto.

[8] K. Soe and M. Myint Sein, "3D virtual view reconstruction from 2D images," in Proc. International Conference of Business and Industrial Research (ICBIR 2010), Bangkok, Thailand, March 2010, pp. 201-204.

[9] R. Koch, "3-D scene modelling from stereoscopic image sequences," Euro-pean Workshop on Combined real and synthetic image processing for broadcast and video productions, pp. 23-24, 1994, Hamburg, Germany.

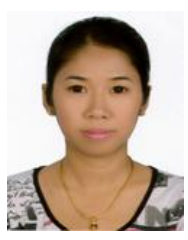

Sandar Aung received the B.C.Tech, B.C.Tech (Hons:) and M.C.Tech degrees in Computer Technology from University of Computer Studies, Yangon, Myanmar, in 2004 , 2005 and 2009, respectively. She is a Ph.D candidate of University of Computer Studies, Mandalay, Myanmar. Her research interests are in the areas of image processing and $3 \mathrm{D}$ shape reconstruction. She is a student member of IEEE. 